\title{
The Diagnostic Value of Plasma miRNA-497, cTnl, FABP3 and GPBB in Pediatric Sepsis Complicated with Myocardial Injury
}

\author{
Chengjiao Huang* \\ Shuna Xiao* \\ Zhi Xia \\ Ying Cheng \\ Yong $\mathrm{Li}$ \\ Wen Tang \\ Buyun Shi \\ Chenguang Qin \\ Hui Xu
}

Department of PICU, Maternal and Child Health Hospital of Hubei Province (Women and Children's Hospital of Hubei Province), Wuhan, People's

Republic of China

*These authors contributed equally to this work
Correspondence: Hui Xu

Department of PICU, Maternal and Child Health Care Hospital of Hubei Province, No. 745 Wuluo Road, Hongshan District, Wuhan, Hubei, 430070, People's Republic of China

Tel +86-027-87169353

Email hbsfy2020picu@I63.com
Objective: To investigate the diagnostic value of plasma miRNA-497, cardiac troponin I (cTnI), fatty acid binding protein 3 (FABP3), glycogen phosphorylase isoenzyme BB (GPBB) in pediatric sepsis complicated with myocardial injury.

Methods: From August 2018 to February 2020, 82 children with sepsis admitted to our hospital and 50 health children who came for physical examination (defined as control group) were enrolled in this study. Children with sepsis and myocardial injury were enrolled in the combined group $(n=35)$, and those without myocardial injury were enrolled in the sepsis group $(n=47)$. General data of three groups were collected, and the levels of miRNA-497, FABP3, GPBB, creatine kinase isoenzyme MB (CK-MB), procalcitonin (PCT), C-reactive protein (CRP), cTnI and N-terminal pro-B-type natriuretic peptide (NT-proBNP) were detected and the cardiac function was measured. The diagnostic value of plasma miRNA-497, cTnI, FABP3 and GPBB in pediatric sepsis complicated with myocardial injury was analyzed.

Results: The infection site of the combined group was not significantly different from that of the sepsis group. The levels of miRNA-497, FABP3, GPBB, CK-MB, PCT, CRP, cTnI, NT-proBNP in the combined group were all higher than those in the sepsis group and the control group $(P<0.05)$, and the left ventricular ejection fraction $(\mathrm{LVEF})$ in the combined group was significantly lower than that in the other two group $(P<0.05)$. The area under the curve (AUC) of the combination of miRNA-497, FABP3, GPBB, and cTnI in the diagnosis of sepsis complicated with myocardial injury was significantly higher than that of CK-MB, PCT, CRP, NT-proBNP alone $(P<0.05)$, but there was no significant difference when compared with miRNA-497, FABP3, GPBB and cTnI alone $(P>0.05)$. When the optimal thresholds of miRNA-497, FABP3, GPBB, and cTnI were set to $2.03,6.23 \mathrm{ng} / \mathrm{mL}, 4.01 \mathrm{ng} / \mathrm{mL}, 1.23 \mathrm{ng} / \mathrm{mL}$, respectively, the sensitivity was $95.65 \%, 88.89 \%$, $82.61 \%, 87.50 \%$, respectively; the specificity was $83.33 \%, 94.12 \%, 83.33 \%, 90.91 \%$, respectively; and the accuracy was $91.43 \%, 91.43 \%, 82.86 \%, 88.57 \%$, respectively. Pearson correlation analysis indicating that miRNA-497 was positively correlated with the levels of FABP3, GPBB, and cTnI in the combined group ( $\mathrm{r}=0.821,0.621,0.782, P<0.05)$.

Conclusion: Plasma miRNA-497, cTnI, FABP3, and GPBB levels were increased in pediatric sepsis complicated with myocardial injury, and their combination had high diagnostic value, which was of great clinical significance for early diagnosis and early treatment of pediatric sepsis complicated with myocardial injury.

Keywords: miRNA-497, cTnI, FABP3, GPBB, pediatric sepsis, myocardial injury, diagnosis

\section{Introduction}

Sepsis refers to the life-threatening organ dysfunction caused by the body's maladjusted response to infection. With high morbidity and mortality, sepsis is the most 
important cause of death for patients in intensive care units. $^{1,2}$ The most common complications of sepsis are myocardial injury and cardiac dysfunction, ${ }^{3}$ about $40 \%$ of patients with sepsis are accompanied by myocardial injury, and its mortality rate is as high as $90 \%{ }^{4}$ Myocardial insufficiency and cardiomyopathy are the main causes of death in children with sepsis, ${ }^{5}$ while the essence of clinical and laboratory diagnosis of myocardial injury is the selection and application of myocardial injury markers. ${ }^{6}$ Therefore, early diagnosis and early treatment are of great clinical value for patients with sepsis complicated with myocardial injury.

At present, there has been no definite and uniform diagnostic criteria for sepsis complicated with myocardial injury. Biomarker detection has a certain value in the diagnosis of cardiac dysfunction in children with sepsis and has become the research direction of laboratory diagnosis of pediatric sepsis complicated with myocardial injury.

Cardiac troponin I (cTnI) is a biomarker of myocardial injury and is widely used in the diagnosis of viral myocarditis and acute myocardial infarction. Huo et $\mathrm{al}^{7}$ confirmed that cTnI had important diagnostic value in the minor myocardial injury after distal coronary artery occlusion. Glycogen phosphorylase isoenzyme BB (GPBB) is the key enzyme for glycogen decomposition in the process of oxidative phosphorylation of cardiomyocytes. Tian ${ }^{8}$ et al confirmed that GPBB had certain value in the diagnosis of myocardial ischemic injury in neonates with asphyxia. Cardiac fatty acid binding protein 3 (FABP3) is a newly discovered highly specific marker of myocardial injury, and it will enter the blood after myocardial cell injury. Ye et $\mathrm{al}^{9}$ confirmed that FABP3 had high diagnostic value in early myocardial infarction caused by early myocardial infarction and acute coronary syndrome. MiRNA is a kind of singlestranded small non-coding RNA, which is closely related to a variety of biological processes such as cell proliferation, differentiation, and apoptosis, and has a certain correlation with the occurrence and development of sepsis. A recent study has confirmed ${ }^{10}$ that the expression of miRNA-497 is abnormal in patients with myocardial infarction, which is likely to become a biomarker of myocardial infarction. However, there have been no clinical reports on miRNA-497, cTnI, FABP3, GPBB in the diagnosis of pediatric sepsis complicated with myocardial injury. Based on this, this study will explore the diagnostic significance of miRNA, cTnI, FABP3 and GPBB in pediatric sepsis complicated with myocardial injury, in order to provide evidence for the early diagnosis and treatment of pediatric sepsis complicated with myocardial injury.

Based on this, the prospective trial was carried to investigate the diagnostic value of plasma miRNA-497, cardiac troponin I (cTnI), fatty acid binding protein 3 (FABP3), glycogen phosphorylase isoenzyme BB (GPBB) in pediatric sepsis complicated with myocardial injury.

\section{Materials and Methods General Data}

From August 2018 to February 2020, the clinical data of 82 children with sepsis admitted to Hubei Maternal and Child Health Hospital were retrospectively analyzed. They were divided into two groups according to whether they were complicated with myocardial injury. The combined group included 35 children with sepsis and myocardial injury, the sepsis group included 47 children with only sepsis. Fifty healthy children who came to our hospital for physical examination during the same period were enrolled in the control group.

This study was approved by the Ethics Committee of Hubei Maternal and Child Health Hospital (approval number: 2020IECLW055), and the guardians of all subjects signed informed consent. This study was conducted in accordance with the Helsinki Declaration of the World Medical Association.

\section{Inclusion and Exclusion Criteria}

Inclusion criteria: (1) The diagnostic criteria for sepsis was based on the "Expert Consensus on the Diagnosis and Treatment of Septic Shock (Infectious Shock) in Children (2015 Edition)"; ; ${ }^{11}$ (2) the diagnostic criteria for myocardial injury was left ventricular ejection fraction (LVEF) $<50 \%$ or $\mathrm{cTnI} \geq 0.15 \mathrm{ng} / \mathrm{mL} ;{ }^{12}$ (3) Absence of myocardial injury at admission in children with sepsis; (4) complete clinical data; (5) the guardians of all subjects signed informed consent.

Exclusion criteria: (1) hematopathy; (2) liver and kidney dysfunction; (3) cardiac insufficiency; (4) immunodeficiency and congenital malformations; (5) long-term use of immunosuppressants or hormone drugs; (6) inherited metabolic diseases; (7) children with poor compliance; (8) malignant tumors. 


\section{Index Detection}

Plasma miRNA-497 Collection and Detection

For each patient, $3-5 \mathrm{~mL}$ fasting ( $>12$ hours) cubital venous blood was extracted and placed into an EDTA anticoagulant tube. After centrifugation at $1500 \mathrm{rpm} / \mathrm{min}$ for $5 \mathrm{~min}$, the plasma was separated and $1 \mathrm{~mL}$ miRNeasy reagent was added into the plasma. The total miRNA was extracted according to the instructions of the miRNeasy plasma extraction kit. ABI 7500 fluorescent quantitative PCR instrument was used for real-time fluorescent quantitative polymerase chain reaction (RT-PCR). The number of cycles for the fluorescence signal in each reaction system to reach the set threshold was the $\mathrm{Ct}$ value, the internal reference was U6. The $2^{-\Delta \Delta \mathrm{Ct}}$ method was used to calculate the relative expression level of the miRNA-497, $\Delta \mathrm{Ct}=\mathrm{Ct}_{\text {target gene }}-\mathrm{Ct}_{\mathrm{u} 6}$.

\section{Detection of Other Laboratory Index}

A total of $8 \mathrm{~mL}$ of cubital venous blood from all the included children was collected on an empty stomach for more than 12 hours the next morning after admission. The blood was evenly divided into 4 samples after anticoagulation treatment. After centrifugation, the supernatant was taken. The levels of FABP3, GPBB, and serum creatine kinase isoenzymes (CK-MB) were detected by enzyme-linked immunosorbent assay (ELISA), and the kits were purchased from the Shenzhen Kangshengbao Biotechnology Co., Ltd. The PCT, CRP, cTnI, NT-proBNP were detected by Hitachi 7080 series automatic biochemical analyzer and SSJ-2-2 multifunctional immunoassay instrument.

\section{Cardiac Function Determination}

The LVEF was evaluated by IU22 color Doppler echocardiography (Philips, the Netherlands). The detection frequency was $2.0 \sim 4.0 \mathrm{MHz}$. The same cardiac cycle was taken as a parameter and the mean value of three cardiac cycles was calculated. All the above operations were performed by attending physicians who have worked in our hospital for more than 8 years.

\section{Statistical Methods}

All data in this study were analyzed using SPSS 23.0 software. Normally distributed measurement data were expressed as mean \pm standard deviation (SD). One-way analysis of variance was used for comparison among the three groups; the pairwise comparison between two groups was performed by the Least-Significant Difference (LSD) test. The categorical data was expressed as rate (\%), and the chi-square test was used for comparison among three groups. Pearson linear correlation analysis or Spearman rank correlation analysis was used for correlation analysis. $P<0.05$ was considered statistically significant.

\section{Results}

\section{Comparison of Demographics and Clinical Characteristics}

In the combined group, there were 23 males and 12 females, their ages were from 3 to 13 years old, with an average age of $6.93 \pm 0.53$ years. In the sepsis group, there were 29 males and 18 females; their ages were from 3 to 12 years old, with an average age of $6.89 \pm 0.47$ years. In the control group, there were 29 males and 21 females; their ages were from 3 to 13 years old, with an average age of $6.92 \pm 0.48$ years.

There was no significant difference in gender and age among the three groups $(P>0.05)$. Compared with the sepsis group, the infection site of the combined group was not significantly different $(P>0.05)$, as shown in Table 1 .

\section{Comparison of miRNA-497, FABP3, GPBB, CK-MB, PCT, CRP, cTnl, NT-proBNP Levels and LVEF Among the Three Groups}

The levels of miRNA-497 expression, FABP3, GPBB, CK-MB, PCT, CRP, cTnI and NT-proBNP in the combined group were all significantly higher than those in the sepsis group and the control group, and LVEF in the combined group was significantly lower than that in the sepsis group and the control group $(P<0.05)$. The levels of miRNA-497 expression, FABP3, GPBB, CK-MB, PCT, CRP, cTnI and NT-proBNP in the sepsis group were all significantly higher than those in the control group, and LVEF in the sepsis group was significantly lower than that in the control group $(P<0.05)$, as shown in Table 2 .

\section{The Diagnostic Value of miRNA-497, FABP3, GPBB and cTnl in Myocardial Injury of Children in the Combined Group}

The AUC value of the combination of miRNA-497, FABP3, GPBB, and cTnI in the diagnosis of sepsis complicated with myocardial injury was significantly higher than that of CK-MB, PCT, CRP, NT-proBNP alone $(P<0.05)$, but there was no significant difference when compared with miRNA-497, FABP3, GPBB and cTnI alone $(P>0.05)$. When the optimal thresholds of miRNA- 
Table I Comparison of Clinical Characteristics Among Three Groups

\begin{tabular}{|c|c|c|c|c|}
\hline Item & Combined Group $(n=35)$ & Sepsis Group $(n=47)$ & Control Group $(n=50)$ & $P$ value \\
\hline Gender (male/female) & $23 / 12$ & $29 / 18$ & $29 / 18$ & 0.915 \\
\hline Age (years old) & $6.93 \pm 0.53$ & $6.89 \pm 0.47$ & $6.92 \pm 0.48$ & 0.925 \\
\hline Infection site $n,(\%)$ & & & & 0.470 \\
\hline Pulmonary infection & $17(48.57)$ & $19(40.43)$ & 0 & \\
\hline Abdominal infection & II (3I.43) & $12(25.53)$ & 0 & \\
\hline Skin and soft tissue infection & I (2.86) & $3(6.38)$ & 0 & \\
\hline Urinary infection & I (2.86) & $2(4.26)$ & 0 & \\
\hline Intracranial infection & $4(11.43)$ & I (2.13) & 0 & \\
\hline Hematogenous infections & $5(14.29)$ & $2(4.26)$ & 0 & \\
\hline
\end{tabular}

Table 2 Comparison of miRNA-497, FABP3, GPBB, CK-MB, PCT, CRP, cTnl, NT-proBNP Levels and LVEF Among the Three Groups

\begin{tabular}{|c|c|c|c|c|}
\hline Index & Combined Group $(n=35)$ & Sepsis Group $(n=47)$ & Control Group $(n=50)$ & $P$ value \\
\hline miRNA-497 & $4.21 \pm 0.73^{* \#}$ & $\mathrm{I} .7 \mathrm{I} \pm 0.7 \mathrm{I}^{*}$ & $1.02 \pm 0.23$ & $<0.001$ \\
\hline FABP3 (ng/mL) & $34.52 \pm 2.45 * \#$ & $13.69 \pm 1.98 *$ & $4.21 \pm 0.43$ & $<0.001$ \\
\hline GPBB (ng/mL) & $22.89 \pm 2.39 * \#$ & $9.23 \pm 1.23 *$ & $2.67 \pm 0.24$ & $<0.001$ \\
\hline CK-MB (U/L) & $44.93 \pm 3.92{ }^{* \#}$ & $19.98 \pm 2.31 *$ & $9.12 \pm 0.32$ & $<0.001$ \\
\hline PCT $(\mu g / L)$ & $17.92 \pm 32.9 * \#$ & $12.23 \pm 0.34^{*}$ & $0.21 \pm 0.08$ & $<0.001$ \\
\hline CRP (mg/L) & $163.29 \pm 12.39$ *\# & $141.29 \pm 14.8^{*}$ & $12.09 \pm 1.03$ & $<0.001$ \\
\hline $\mathrm{cTnl}(\mathrm{ng} / \mathrm{mL})$ & $4.18 \pm 0.38^{* \#}$ & $0.08 \pm 0.01 *$ & $0.03 \pm 0.01$ & $<0.001$ \\
\hline NT-proBNP (ng/L) & $17.93 \pm 1.29 * \#$ & $12.19 \pm 2.13^{*}$ & $6.23 \pm 0.21$ & $<0.001$ \\
\hline LVEF (\%) & $46.12 \pm 4.39 * \#$ & $61.29 \pm 5.83 *$ & $67.09 \pm 2.09$ & $<0.001$ \\
\hline
\end{tabular}

Notes: *Compared with control group, $P<0.05 ;{ }^{\#}$ Compared with sepsis group, $P<0.05$.

Abbreviations: FABP3, fatty acid binding protein 3; GPBB, glycogen phosphorylase isoenzyme BB; CK-MB, creatine kinase isoenzyme MB; PCT, procalcitonin; CRP, C-reactive protein; cTnl, cardiac troponin I; NT-proBNP, N-terminal B-type natriuretic peptide; LVEF, left ventricular ejection fraction.

497, FABP3, GPBB, and cTnI were set to $2.03,6.23 \mathrm{ng} /$ $\mathrm{mL}, 4.01 \mathrm{ng} / \mathrm{mL}, 1.23 \mathrm{ng} / \mathrm{mL}$, their sensitivity was $95.65 \%$, $88.89 \%, 82.61 \%, 87.50 \%$, respectively; their specificity was $83.33 \%, 94.12 \%, 83.33 \%, 90.91 \%$, respectively; and their accuracy was $91.43 \%, 91.43 \%, 82.86 \%, 88.57 \%$, respectively, as shown in Table 3 . Sensitivity $=$ true positive/(true positive + false negative) $\times 100 \%$, specificity $=$ true negative/(false positive + true negative) $\times 100 \%$, accuracy $=($ true positive + true negative $) / \mathrm{n} \times 100 \%$.

\section{Correlation Analysis Between} miRNA-497 and FABP3, GPBB, cTnl Levels in the Combined Group

According to Pearson correlation analysis, the expression of miRNA-497 in the combined group was positively correlated with the levels of FABP3, GPBB, and cTnI $(\mathrm{r}=0.821,0.621,0.782, P<0.05)$, which suggested that miRNA-497 was well correlated with FABP3, GPBB and cTnI, and sepsis complicated with myocardial injury was closely correlated with miRNA-497 and FABP3, GPBB and cTnI.

\section{Discussion}

The main findings of this study are 1) The levels of miRNA497, FABP3, GPBB, CK-MB, PCT, CRP, cTnI, NT-proBNP in the combined group were all higher than those in the sepsis group and the control group, and the left ventricular ejection fraction (LVEF) in the combined group was significantly lower than that in the other two groups, 2) The area under the curve (AUC) of the combination of miRNA-497, FABP3, GPBB, and cTnI in the diagnosis of sepsis complicated with myocardial injury was significantly higher than 
Table 3 The Diagnostic Value of miRNA-497, FABP3, GPBB and cTnl in Myocardial Injury of the Combined Group

\begin{tabular}{|l|c|c|c|c|c|}
\hline Index & $\begin{array}{c}\text { Optimal Threshold } \\
\text { Value }\end{array}$ & AUC (95\% Cl) & $\begin{array}{c}\text { Sensitivity } \\
\text { (\%) }\end{array}$ & $\begin{array}{c}\text { Specificity } \\
\text { (\%) }\end{array}$ & $\begin{array}{c}\text { Accuracy } \\
\text { (\%) }\end{array}$ \\
\hline miRNA-497 & 2.03 & $0.916(0.849-0.980)$ & 95.65 & 83.33 & 91.43 \\
\hline FABP3 $(\mathrm{ng} / \mathrm{mL})$ & 6.23 & $0.912(0.862-0.963)$ & 88.89 & 94.12 & 91.43 \\
\hline GPBB $(\mathrm{ng} / \mathrm{mL})$ & 4.01 & $0.904(0.887-0.973)$ & 82.61 & 83.33 & 82.86 \\
\hline cTnl $(\mathrm{ng} / \mathrm{mL})$ & 1.23 & $0.838(0.793-0.864)$ & 87.50 & 90.91 & 88.57 \\
\hline CK-MB $(\mathrm{U} / \mathrm{L})$ & 11.09 & $0.701(0.623-0.863)$ & 61.11 & 64.71 & 62.86 \\
\hline PCT $(\mu \mathrm{g} / \mathrm{L})$ & 0.98 & $0.809(0.763-0.839)$ & 72.22 & 88.23 & 80.00 \\
\hline CRP $(\mathrm{mg} / \mathrm{mL})$ & 14.82 & $0.726(0.669-0.762)$ & 62.50 & 78.95 & 71.43 \\
\hline NT-proBNP $(\mathrm{ng} / \mathrm{L})$ & 7.34 & $0.804(0.776-0.832)$ & 72.22 & 82.35 & 77.14 \\
\hline $\begin{array}{l}\text { Combination of miRNA-497, FABP3, GPBB, } \\
\text { and cTnl }\end{array}$ & & $0.953(0.896-0.997)$ & 96.43 & 85.71 & 91.43 \\
\hline
\end{tabular}

Notes: *Compared with CK-MB, PCT, CRP, NT-proBNP alone, $P<0.05$.

Abbreviations: FABP3, fatty acid binding protein 3; GPBB, glycogen phosphorylase isoenzyme BB; cTnl, cardiac troponin I; CK-MB, creatine kinase isoenzyme MB; PCT, procalcitonin; CRP, C-reactive protein; NT-proBNP, N-terminal B-type natriuretic peptide; LVEF, left ventricular ejection fraction.

that of CK-MB, PCT, CRP, NT-proBNP alone $(P<0.05)$, but there was no significant difference when compared with miRNA-497, FABP3, GPBB and cTnI alone, 3) MiRNA497 was positively correlated with the levels of FABP3, GPBB, and $\mathrm{cTnI}$ in the combined group.

In recent years, the morbidity and mortality of pediatric sepsis have been on the rise, and the main cause of death of pediatric sepsis is myocardial injury, which is one of the complications of sepsis. In clinical practice, the early symptoms of myocardial injury in some children with sepsis are not obvious. Therefore, how to diagnose myocardial injury as soon as possible is one of the important clinical issues that needs to be solved urgently. At present, the clinically commonly used myocardial indicators include cTnI, CK-MB, NT-proBNP and echocardiography. However, they all have certain limitations, which is not conducive to clinical promotion. Therefore, it is of great value to find new markers for early diagnosis of myocardial injury in children with sepsis.

MiRNA is a type of non-coding RNA that has been newly studied in recent years and can regulate a variety of biological functions. More and more studies have confirmed $^{13,14}$ that miRNA is closely related to the occurrence and development of a variety of cardiovascular diseases, including arrhythmia, hypertension, myocardial infarction, myocardial fibrosis, and myocardial hypertrophy. Maciejak et $\mathrm{al}^{15}$ confirmed that the expression levels of some miRNAs in peripheral blood or plasma of patients with myocardial injury were significantly increased, and miRNAs may become a novel marker for early diagnosis of myocardial injury. However, there are few reports on whether expression of miRNA-497 in plasma can be used as a molecular marker for the early diagnosis of sepsis complicated with myocardial injury.

cTnI is a structural protein of cardiomyocytes, which can be used to evaluate the degree of damage or necrosis of cardiomyocytes, which has high specificity. Its physiological function is mainly to react with actin and myosin under the mediation of calcium ion, and maintain the relaxation and contraction of myocardium. Li et al ${ }^{16}$ confirmed that after myocardial injury, cTnI was decomposed from tropomyosin and released into the blood and circulated throughout the body along with the blood, which had a long window period, so the myocardial injury within 6 hours cannot be diagnosed in time by cTnI.

GPBB is composed of 843 amino acids, which are highly expressed in cardiomyocytes and can provide energy for the myocardial contraction. Under normal circumstances, the sarcoplasmic reticulum combines with cardiomyocytes containing GPBB, and GPBB is released into the blood by the cardiomyocytes when myocardial injury occurs. Research by $\mathrm{Li}$ et $\mathrm{al}^{17}$ confirmed that the blood GPBB level rose rapidly when the body developed heart diseases such as myocardial infarction or myocardial 
ischemia injury, indicating that GPBB can be used as a marker for myocardial injury.

FABP3 is an acidic protein composed of 132 amino acid residues, which is specific in myocardial tissue. FABP3 combines with long-chain fatty acid to transport cytoplasm and decompose and transform the energy needed by myocardium. The relative molecular mass of FABP3 is only $15,000 \mathrm{Da}$. In the early stage of myocardial injury, fatty acid function will increase when myocardial ischemia and hypoxia occur, which increase the level of FABP3 in cardiomyocytes. At the same time, myocardial ischemia and hypoxia will increase the permeability of cell membranes, and FABP3 in the cytoplasm quickly penetrates the cell membrane and enters the intercellular substance due to its small relative molecular mass, increasing the level of FABP3 in the blood. ${ }^{18}$ Gerede et $\mathrm{al}^{19}$ confirmed that the level of FABP3 in the blood was significantly increased after $0-3$ hours of myocardial injury, and gradually returned to the normal level after 12-24 hours. Therefore, FABP3 level in blood can be used to diagnose early myocardial injury, and has higher specificity and sensitivity than traditional myocardial markers.

The results of this study showed that the levels of miRNA-497, FABP3, GPBB, CK-MB, PCT, CRP, cTnI, and NT-proBNP in the combined group were significantly higher than those in the sepsis group and the control group, and the LVEF in the combined group was significantly lower than that in the sepsis group and the control group. The levels of miRNA-497, FABP3, GPBB, CKMB, PCT, CRP, cTnI, and NT-proBNP in the sepsis group were significantly higher than those in the control group, and the LVEF in the sepsis group was significantly lower than that in the control group. These results suggested that plasma miRNA-497, FABP3, GPBB, CK-MB, PCT, CRP, cTnI and NT-proBNP levels in children with sepsis complicated with myocardial injury were significantly higher than those in children with only sepsis, and plasma miRNA-497, FABP3, GPBB, CK-MB, PCT, CRP, cTnI and NT-proBNP levels in children with sepsis were significantly higher than those in healthy children. This may be because plasma miRNA-497, FABP3, GPBB, CK-MB, PCT, CRP, cTnI, and NT-proBNP are involved in the occurrence and development of sepsis and myocardial injury.

The study of Yang et $\mathrm{al}^{20}$ confirmed that the plasma miRNA-499 expression level of patients with sepsis complicated with myocardial injury was significantly higher than that of normal people. Therefore, plasma miRNA-499 can be used as a biological marker for the diagnosis of patients with sepsis complicated with myocardial injury. Boon et $\mathrm{al}^{21}$ showed that miRNA can be produced after myocardial cell injury and released into the blood, which was significantly increased in patients with myocardial infarction and was closely related to the degree of myocardial injury. Research by $\mathrm{Wu}$ et $\mathrm{al}^{22}$ confirmed that 24 hour dynamic electrocardiogram combined with $\mathrm{cTnI}$ can be used to evaluate radiation-induced cardiac injury induced by thoracic tumor radiotherapy. Sheyin et $\mathrm{al}^{23}$ confirmed that cTnI had high specificity and sensitivity, and it occurred early after onset and lasted for a long time, which can be used as an effective biomarker for the diagnosis of sepsis complicated with myocardial injury. The study of Qi et $\mathrm{al}^{24}$ confirmed that H-FABP and NTprobNP can be used for the early diagnosis of sepsis complicated with myocardial injury, and had important guiding value for clinical medication. The results of this study showed that the AUC value of the combination of miRNA-497, FABP3, GPBB, and cTnI in the diagnosis of sepsis complicated with myocardial injury was significantly higher than that of CK-MB, PCT, CRP, and NTproBNP alone.

When the optimal thresholds of miRNA-497, FABP3, GPBB, and cTnI were set to $2.03,6.23 \mathrm{ng} / \mathrm{mL}, 4.01 \mathrm{ng} / \mathrm{mL}$, $1.23 \mathrm{ng} / \mathrm{mL}$, their sensitivity was $95.65 \%, 88.89 \%, 82.61 \%$, $87.50 \%$, respectively; their specificity was $83.33 \%$, $94.12 \%, 83.33 \%, 90.91 \%$, respectively; and their accuracy was $91.43 \%, 91.43 \%, 82.86 \%, 88.57 \%$, respectively, suggesting that plasma miRNA-497, FABP3, GPBB, cTnI can be used to effectively diagnose pediatric sepsis complicated with myocardial injury, which has high sensitivity, specificity and accuracy. Therefore, the detection of plasma miRNA-497, FABP3, GPBB and cTnI levels in children with sepsis complicated with myocardial injury has important clinical diagnostic value.

This study further analyzed the correlation between miRNA-497 expression and FABP3, GPBB and cTnI levels in children with sepsis complicated with myocardial injury. According to Pearson correlation analysis, the expression of miRNA-497 was positively correlated with levels of FABP3, GPBB and cTnI in the combined group, suggesting that miRNA-497 had a good correlation with FABP3, GPBB and cTnI in children with sepsis complicated with myocardial injury. Research by Shi et $\mathrm{al}^{25}$ confirmed that the combination of miRNA and other markers of myocardial injury was more effective in diagnosing sepsis complicated with myocardial injury. Research by 
Yang et $\mathrm{al}^{26}$ confirmed that plasma miRNA-1, miRNA208a and miRNA-499 can all be used as effective biomarkers for myocardial injury. Reiter et $\mathrm{al}^{27}$ confirmed that FABP3 had high sensitivity and specificity in evaluating myocardial reperfusion. Research by $\mathrm{Lu}$ et $\mathrm{al}^{28}$ found that GPBB was better than traditional myocardial markers in the early diagnosis of sepsis complicated with myocardial injury. Guo et $\mathrm{al}^{29}$ confirmed that cTnI and IMA can be used as prognostic indicators for patients with sepsis complicated with myocardial injury. It can be concluded that plasma miRNA-497, FABP3, GPBB and cTnI can be used in the diagnosis of pediatric sepsis complicated with myocardial injury, and their combined application had a better diagnosis effect.

There were some limitations in our study as follows: (1) This study was a single-center study, so a multicenter clinical study with larger sample size will be conducted to improve the diagnosis and treatment evaluation system for pediatric sepsis complicated with myocardial injury. (2) The specific mechanism of miRNA-497, FABP3, GPBB and cTnI in the occurrence and development of sepsis complicated with myocardial injury is still unclear.

\section{Conclusion}

In conclusion, plasma miRNA-497, FABP3, GPBB, and cTnI were all involved in the pathogenesis of pediatric sepsis complicated with myocardial injury, and can be used for the early diagnosis of pediatric sepsis complicated with myocardial injury. Therefore, plasma miRNA-497, FABP3, GPBB, and cTnI are expected to be new diagnostic markers for pediatric sepsis complicated with myocardial injury.

\section{Data Sharing Statement}

The datasets generated and analyzed during the current study are available from the corresponding author on reasonable request.

\section{Ethics Approval and Consent to Participate}

The study protocol was approved by the Ethics Committee of Hubei Maternal and Child Health Hospital (approval number: 2020IECLW055). Written informed consent was obtained from all the study subjects' parents before enrollment.

\section{Funding}

No funding was received for this study.

\section{Disclosure}

The authors declare that they have no competing interests.

\section{References}

1. National Taiwan University Hospital Health Economics and Outcome Research Group; Yo CH, Hsu TC, Gabriel Lee MT, et al. Trend and outcome of sepsis in children: a nationwide cohort study. J Paediatr Child Health. 2018;54(7):776-783. doi:10.1111/jpc.13849.

2. Duan Y, Jin D, Xu Y, et al. [Risk factors and prognosis of acute kidney injury in children with sepsis in pediatric intensive care unit]. Zhonghua Wei Zhong Bing Ji Jiu Yi Xиe. 2019;31(8):1004-1007. Chinese. doi:10.3760/cma.j.issn.2095-4352.2019.08.018

3. Chen FC, Xu YC, Zhang ZC. Research progress on the diagnostic value of new biochemical markers in septic myocardial injury. Chin Gen Pract. 2021;668(05):30-35+42.

4. Peters C, Kissoon N. Surviving sepsis in children: our job is only half done. Pediatr Crit Care Med. 2019;20(6):568-569. doi:10.1097/ PCC.0000000000001909

5. Sun Y, Tang H, Zhang Y. The value of serum FABP3 combined with GPBB in the diagnosis of myocardial injury in children with sepsis. Shandong Med. 2019;059(004):55-58.

6. Shi MQ, Su FF, Li JX, et al. Status quo of the diagnosis and treatment of myocardial injury of children with sepsis. Prog Modern Biomed. 2017;17(14):2789-2792.

7. Huo YF, Ma MF, Tian HS. Comparison of the diagnostic value of hs-cTnI and cTnI in MMD after distal coronary occlusion. J Clin Exp Med. 2019;285(05):51-54.

8. Tian B, Liu LF, Deng HZ, et al. The level of glycogen phosphorylase isoenzyme BB and ischemia modified albumin in newborn with asphyxia and myocardial injury and its clinical significance. Int J Lab Med. 2017;38(17):2342-2343, 2346.

9. Ye XD, He Y, Wang S, et al. Heart-type fatty acid binding protein (H-FABP) as a biomarker for acute myocardial injury and long-term post-ischemic prognosis. Acta Pharmacol Sin. 2018;39 (7):1155-1163. doi:10.1038/aps.2018.37

10. Li Z, Lu J, Luo Y, et al. High association between human circulating microRNA-497 and acute myocardial infarction. Sci World J. 2014;2014:931845.

11. The Subspecialty Group of Emergency, the Society of Pediatrics, Chinese Medical Association; the Subspecialty Group of Pediatrics, the Society of Emergency, Chinese Medical Association; the Subspecialty Group of Pediatric Critical Care Physicians, China Physicians Association. $J$ Appl Clin Pediatr. 2015;30 (22):1687-1691.

12. Corsten MF, Dennert R, Jochems S, et al. Circulating microRNA-208b and microRNA-499 reflect myocardial damage in cardiovascular disease. Circ Cardiovasc Genet. 2010;3(6):499-506. doi:10.1161/CIRCGENETICS.110.957415

13. Ge C, Liu J, Dong S. miRNA-214 protects sepsis-induced myocardial injury. Shock. 2018;50(1):112-118. doi:10.1097/SHK.0000 000000000978

14. Kim EN, Kim CJ, Kim SR, et al. High serum CRP influences myocardial miRNA profiles in ischemia-reperfusion injury of ratheart. PLoS One. 2019;14(5):e0216610. doi:10.1371/journal. pone. 0216610

15. Maciejak A, Kiliszek M, Opolski G, et al. miR-22-5p revealed as a potential biomarker involved in the acute phase of myocardial infarction via profiling of circulating microRNAs. Mol Med Rep. 2016;14(3):2867-2875. doi:10.3892/mmr.2016.5566 
16. Li HX, Liu ZM, Zhao SJ, et al. Measuring both procalcitonin and C-reactive protein for a diagnosis of sepsis in critically ill patients. J Int Med Res. 2014;42(4):1050-1059. doi:10.1177/ 0300060514528483

17. $\mathrm{Li} \mathrm{RB}, \mathrm{Li}$ YF. Detection and clinical significance of plasma cardiotrophin-1 and glycogen phosphorylase isoenzyme BB in asphyxiated newborn infants with myocardial injury. Pract Clin Med. 2014;15(6):86-88, 90.

18. Inönü H, Acu B, Pazarlı AC, et al. The value of the computed tomographic obstruction index in the identification of massive pulmonary thromboembolism. Diagn Interv Radiol. 2012;18 (3):255-260. doi:10.4261/1305-3825.DIR.4597-11.4

19. Gerede DM, Güleç S, Kiliçkap M, et al. Comparison of a qualitative measurement of heart-type fatty acid-binding protein with other cardiac markers as an early diagnostic marker in the diagnosis of non-ST-segment elevation myocardial infarction. Cardiovasc J Afr. 2015;26(6):204-209. doi:10.5830/CVJA-2015-028

20. Yang YL, He J, Xiao ZY. The diagnostic value of microRNA-499 for myocardial injury in patients with sepsis. Chin Crit Care Med. 2015;027(003):218-220.

21. Boon RA, Dimmeler S. MicroRNAs in myocardial infarction. Nat Rev Cardiol. 2015;12(3):135-142. doi:10.1038/nrcardio.2014.207

22. Wu X, Yang QH, Liu XJ, et al. Application of 24-hour dynamic electrocardiogram combined with cardiac troponin I in evaluating radiation-induced cardiac injury. Chin J Radiat Oncol. 2020;29 (06):421-426.
23. Sheyin O, Davies O, Duan W, et al. The prognostic significance of troponin elevation in patients with sepsis: a meta-analysis. Heart Lung. 2015;44(1):75-81. doi:10.1016/j.hrtlng.2014.10.002

24. Qi HN, Zhang JJ, He JQ, Wang WZ, Li YQ. Roles for heart-type fatty acid-binding protein and $\mathrm{N}$-terminal probrain natriuretic peptide in myocardium injury in sepsis. Chin Gen Pract. 2017;20 (9):1042-1048.

25. Shi MQ, Su FF, Li JX, et al. Research in clinical applications value of pediatric sepsis markers of myocardial injury. Chin J Crit Care Med. 2016;36(6):486-489,490.

26. Yang W, Shao J, Bai X, et al. Expression of Plasma microRNA-1/21/ 208a/499in myocardial ischemic reperfusion injury. Cardiology. 2015;130(4):237-241. doi:10.1159/000371792

27. Reiter M, Twerenbold R, Reichlin $T$, et al. Heart-type fatty acid-binding protein in the early diagnosis of acute myocardial infarction. Heart. 2013;99(10):708-714. doi:10.1136/heartjnl-2012303325

28. Lu CF, Chi XM, Li H, et al. The diagnostic value of glycogen phosphorylase isoenzyme $\mathrm{BB}$ in myocardium injury in sepsis. $J$ Clin Emerg Med. 2019;20(12):931-934,940.

29. Guo J, Tang J, Xia YC, et al. Diagnostic significance of brain natriuretic peptide and cardiac troponin I in sepsis induced myocardial dysfunction. Chin J Med Guide. 2014;16(04):726-727+729.

\section{Publish your work in this journal}

Therapeutics and Clinical Risk Management is an international, peerreviewed journal of clinical therapeutics and risk management, focusing on concise rapid reporting of clinical studies in all therapeutic areas, outcomes, safety, and programs for the effective, safe, and sustained use of medicines. This journal is indexed on PubMed Central, CAS,
EMBase, Scopus and the Elsevier Bibliographic databases. The manuscript management system is completely online and includes a very quick and fair peer-review system, which is all easy to use. Visit http://www.dovepress.com/testimonials.php to read real quotes from published authors 